\title{
Review
}

\section{Potential of ultrasound in the evaluation of acute appendicitis during pregnancy: a systematic review and meta-analysis}

\author{
Yunlong $\mathrm{Li}^{1}$, Shikuan $\mathrm{Li}^{1, *} \odot$ \\ ${ }^{1}$ Department of Emergency General Surgery, the Affiliated Hospital of Qingdao University, 266003 Qingdao, Shandong, China \\ *Correspondence: worldwidth@aliyun.com (Shikuan Li) \\ Academic Editor: Michael H. Dahan \\ Submitted: 18 March 2021 Revised: 29 May 2021 Accepted: 8 June 2021 Published: 12 January 2022
}

\begin{abstract}
Objective: To assess the accuracy of ultrasound in diagnosing acute appendicitis in pregnant women. Mechanism: The National Library of Medicine (MEDLINE, 1990-2020), Excerpta Medica Database (EMBASE,1946-2020) and the Cochrane Controlled Trials Register (CENTRAL) were used to extract articles that were published in English. A total of five studies involving 521 patients were selected. The DerSimonian and Laird random-effects model and Quality Assessment Tool for Diagnostic Accuracy (QUADAS-2) were used to analyze the data. Findings in brief: We identified 140 related articles and included 5 articles enrolling 521 patients. The values obtained using ultrasound for appendicitis during pregnancy were sensitivity of 0.62 ( $95 \%$ Confidence interval (CI): $0.43-0.78$ ), the specificity of 0.91 (95\% CI: 0.74-0.97), and the Positive Likelihood Ratio of 7.0 (95\% CI: 2.5-19.7), the Negative Likelihood Ratio of 0.41 (95\% CI: 0.27-0.63) and the Diagnostic Odds Ratio of 17 (95\% CI : 6-49). Conclusion: Ultrasound had medium-level sensitivity and high specificity for the diagnosis of appendicitis in pregnant women.
\end{abstract}

Keywords: Acute appendicitis; Ultrasound; Diagnostic study; Pregnancy; Meta-analysis

\section{Introduction}

Although acute appendicitis in pregnant women is uncommon, it is the primary cause of non-obstetric surgeries with an incidence rate of 1 in 1250-1500 pregnant women [1-4]. Precise diagnosis of appendicitis is challenging as it shares several symptoms which may be considered normal during pregnancy. For example, nausea and vomiting may be recognized as morning sickness, and abdominal pain can be misdiagnosed as ectopic pregnancy. Moreover, the complex physiological processes operating during pregnancy might also make clinical signs of appendicitis obscure. For instance, the increscent uterus may displace appendix superiorly to out of the right lower quadrant in some pregnant women. Furthermore, Lee and his colleagues [5] reported that the rate of negative appendectomy is higher in pregnant women and is associated with increased risk of fetal loss and/or premature birth [6,7]. In addition, negative appendectomy and has no substantial impact on decreasing the rate of perforated appendicitis [8]. Therefore, it is crucial for surgeons to diagnose appendicitis accurately, especially in pregnant women.

Several imaging examinations have improved diagnostic accuracy for appendicitis and have avoided unnecessary appendectomies [9]. Ultrasound has multiple advantages including the lack of destructive and ionizing radiations. In addition, ultrasound is a well-established and widely recommended imaging tool to check for the presence of appendicitis in pregnant women $[10,11]$. However, the diagnostic performance of the ultrasound for acute appendicitis in pregnancy is not well understood. Some stud- ies found that the sensitivity of the ultrasound was between 0.18 [12] and 0.79 [13], while the specificity was from 0.68 [14] to 0.99 [12]. Therefore, this study aims to investigate the sensitivity and specificity of ultrasound for the diagnosis of acute appendicitis in pregnant patients. We hypothesize that ultrasound is a useful test in the diagnosis of acute appendicitis for pregnant patients with medium sensitivity and high specificity.

\section{Materials and methods}

\subsection{Search method}

We performed a systematic computerized search using The National Library of Medicine (MEDLINE), Excerpta Medica Database (EMBASE) and the Cochrane Controlled Trials Register (CENTRAL) databases. The query terms "acute appendicitis", "pregnancy", "diagnosis" and "ultrasound" were used. English articles published up until the end of June 2020 were reviewed. Totally 140 articles were reviewed.

\subsection{Study eligibility and selection}

Original research articles, retrospective studies and prospective studies were reviewed. The studies were selected if it involved pregnant women with suspected acute appendicitis and ultrasound was used as a diagnostic tool. In addition, study selection was based on the availability of FP (false positive), FN (false negative), TP (true positive) and TN (true negative).

The initial assessment of studies was based on the article title, abstract and availability of full text. Exclusion was 
Table 1. Characteristics of included studies.

\begin{tabular}{|c|c|c|c|c|c|c|c|c|c|}
\hline \multirow{2}{*}{ Study ID } & \multirow{2}{*}{ Publication type } & \multirow{2}{*}{ Study type } & \multirow{2}{*}{ Sample size } & \multirow{2}{*}{ Average age } & \multirow{2}{*}{ Risk of bias } & \multirow{2}{*}{\multicolumn{4}{|c|}{$\begin{array}{l}\text { Sonographer } \quad \text { AA } \\
\text { experience prevalence }(\%) \text { sensitivity }(\%) \text { specificity }(\%)\end{array}$}} \\
\hline & & & & & & & & & \\
\hline Yoav 2019 [14] & Full text & Retrospective & 86 & 31.3 & High & Not state & 67 & 69 & 68 \\
\hline Abbas 2015 [16] & Full text & Retrospective & 36 & 27.29 & High & High & 86 & 61 & 80 \\
\hline Lior 2016 [17] & Full text & Retrospective & 66 & 28 & High & Not state & 79 & 65 & 86 \\
\hline James 2018 [13] & Full text & Retrospective & 216 & 25.8 & High & High & 82 & 79 & 92 \\
\hline Joseph 2015 [12] & Full text & Retrospective & 117 & Not available & High & High & 9.4 & 18 & 99 \\
\hline
\end{tabular}

applied for studies involving patients who did not match the review question or ultrasound was not used as a diagnosis tool, or if they were reviews, case reports, or if they did not provide the required data.

\subsection{Quality assessment and data extraction}

The quality and applicability of articles were evaluated by the Quality Assessment Tool for Diagnostic Accuracy (QUADAS-2) [15]. Four assessments including index test, flowing, patient selection, reference standard and timing were analysed.

\subsection{Statistical analysis}

The Review Manager (RevMan) (Version 5.4. The Cochrane Collaboration, 2020, London, United Kingdom) was used to perform the analyses and generation of the forest plot and risk of bias and applicability concerns graphs. Stata (revision 20 Mar 2013 copyright 1985-2001 StataCorp LP, College Station, TX, USA) was also used for evaluating heterogeneity and making summary receiver operating characteristic curve (SROC) curve. The sensitivity and specificity of data were assessed using DerSimonian and Laird random-effects model.

\section{Results}

\subsection{Search results}

140 articles were identified from the initial search and were assessed based on titles and abstracts. Eventually, 5 studies were included for analysis in this study (Fig. 1) [1214,16,17]. The key parameters of included studies are listed in the Table 1 (Ref. [12-14,16,17]).

\subsection{Study characteristics and quality assessment}

The five selected studies were retrospective and involved 521 participants, of which studies involving pregnant women seeking diagnosis of appendicitis were included. All trials were single medical center. Pathological test after appendectomy was used to determine the diagnosis performance of ultrasound and served as the standard reference.

Of the five selected studies, three studies compared the working performance of ultrasound for the diagnosis of acute appendicitis in pregnant versus non-pregnant subjects $[14,16,17]$. One study explored whether performing a delayed repeat of ultrasound could increase the diagnostic yield [13]. Another study compared the diagnosis accuracy between ultrasound and Magnetic Resonance Imaging (MRI) [12].

All selected studies were retrospective studies by obtaining the information from hospital digital records. None of the article used consecutive or random sampling method for selection of patients and all included certain patients (the number of patients ranged from 2 [13] to 25 [14]) who meet the included criteria but had no record for ultrasound. The ultrasound was applied before operation; therefore, results of the index test were deciphered without a reference standard. Four out of five studies $[12,13,16,17]$ mentioned that ultrasound results were interpreted by experienced radiologists. Three studies $[12,13,17]$ gave reasons for categorization using pre-specified thresholds. None of the five studies explained whether the pathology results were interpreted independently of the index tests. All the participants who were applied with ultrasound diagnosis receive the same pathology test.

\subsection{Meta-analysis}

To diagnose appendicitis in pregnancy, the ultrasound sensitivity was 0.62 (95\% CI: $0.43,0.78)$, the specificity was 0.91 (95\% CI: 0.74, 0.97), the Positive Likelihood Ratio was 7.0 (95\% CI: $2.5,19.7)$, the Negative Likelihood Ratio was 0.41 (95\% CI: $0.27,0.63)$ and the Diagnostic Odds Ratio was 17 (95\% CI: 6, 49). Forest plots of sensitivity and specificity and the analysis for SROC were shown in Figs. 2,3 respectively. High variation was observed in the specificity $\left(\mathrm{I}^{2}=82.67 \%\right)$ and sensitivity $\left(\mathrm{I}^{2}=85.75 \%\right)$ of ultrasound in diagnosing appendicitis in pregnant women. The mean AUC was $0.83 \pm 0.03$ and the Q index was 28.07 \pm 4.00 (Fig. 4). And the Deeks' Funnel Plot Asymmetry Test $p$ value was 0.42 (Fig. 5).

\section{Discussion}

Meta-analysis studies [18-20] showed that the sensitivity as well as specificity of the ultrasound for the diagnosis of acute appendicitis were $69 \%-83.1 \%$ and $81 \%$ $93 \%$ respectively. Moreover, ultrasound is widely available, economical, and imposes no adverse ionizing radiation [12]. However, Yoav et al. [14] showed that ultrasound examination of pregnant women might provide an incon- 


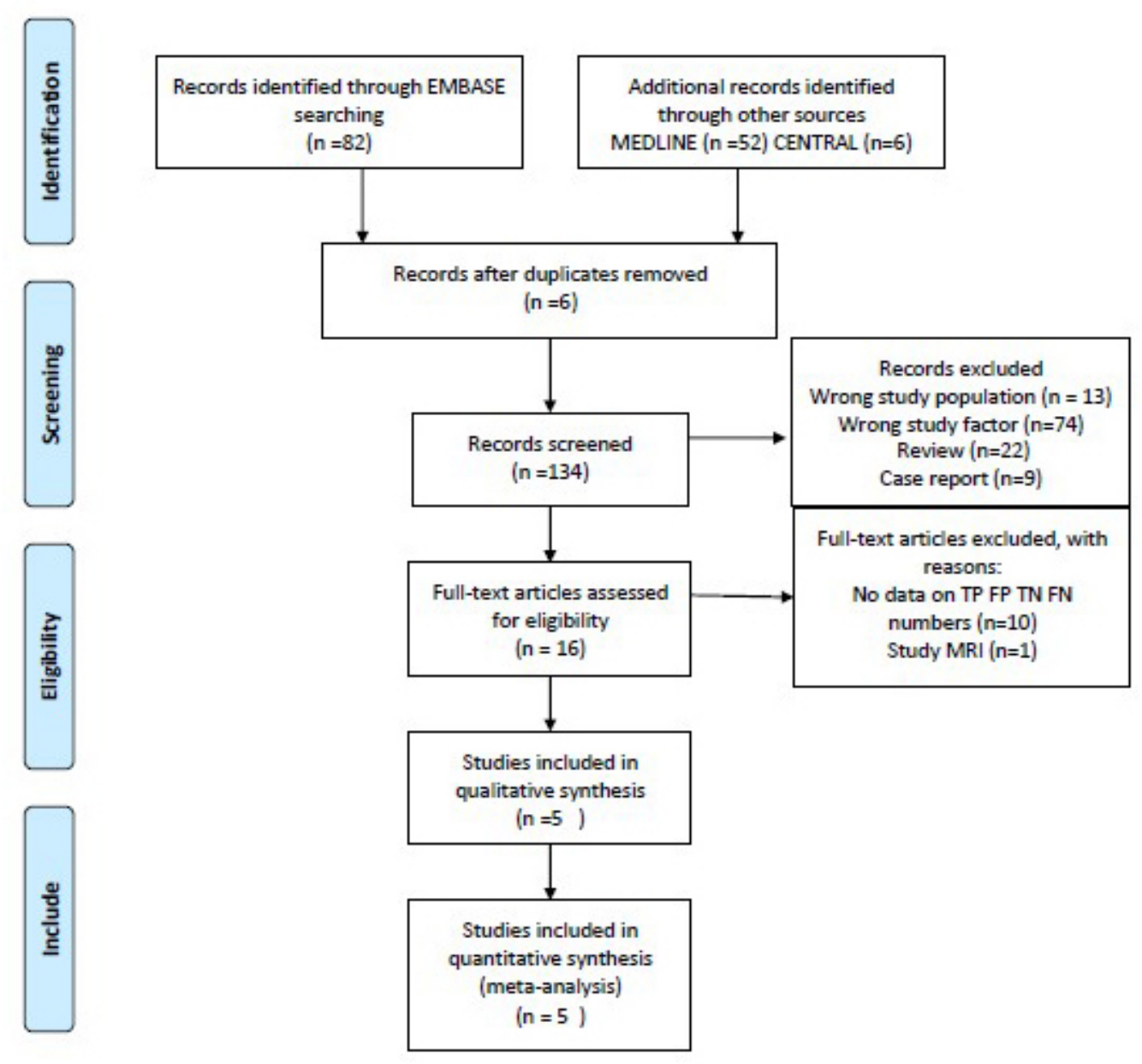

Fig. 1. The process of study selection.

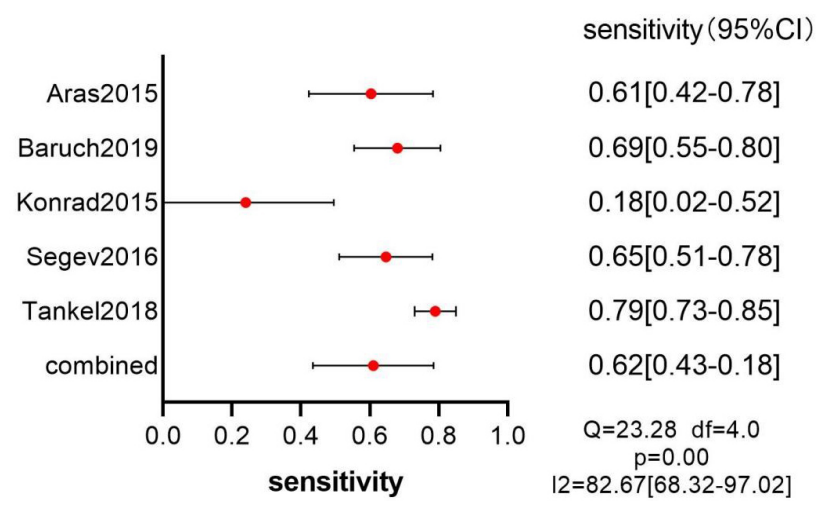

Fig. 2. Forest plot-sensitivity of ultrasound.

clusive data (43.3\% vs. $11.1 \%, p<0.001)$, while Lior and colleagues [17] indicated that the diagnostic performance of ultrasound was similar between the pregnant and nonpregnant patients (AUC 0.76 and 0.73 respectively, $p=$ 0.78).

The sensitivity of CT is $91-98.5 \%$ and the specificity is $90-98 \%$ [21-23]. The excellent performance on diagnosis makes CT the most widely used examination in gen-

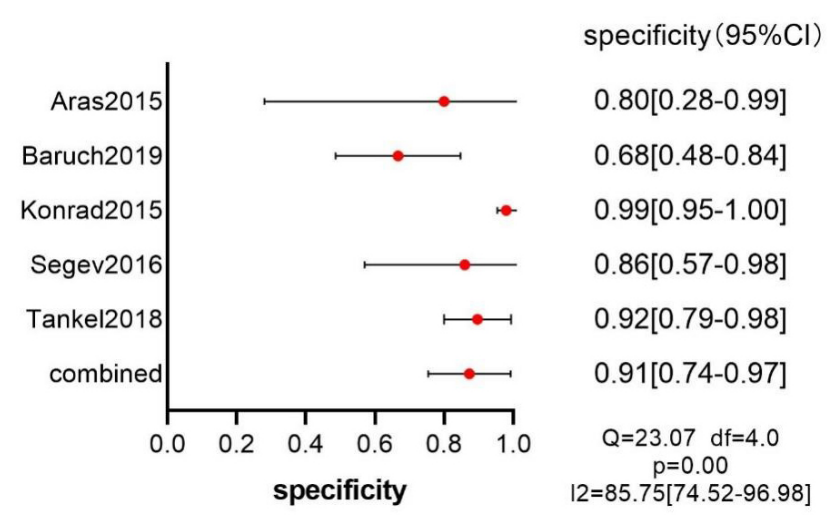

Fig. 3. Forest plot-specificity of ultrasound.

eral population with suspected appendicitis. However, CT is not recommended for pregnant women due to its radiation. The consequence of radiation exposure in fetuses is mostly based on observations rather than original research which is banned due to ethical issues [24].

Kave and his colleagues [25] reported that MRI had sensitivity of $91.8 \%$ whereas the specificity of $97.9 \%$ in diagnosing appendicitis in pregnant women. According to 


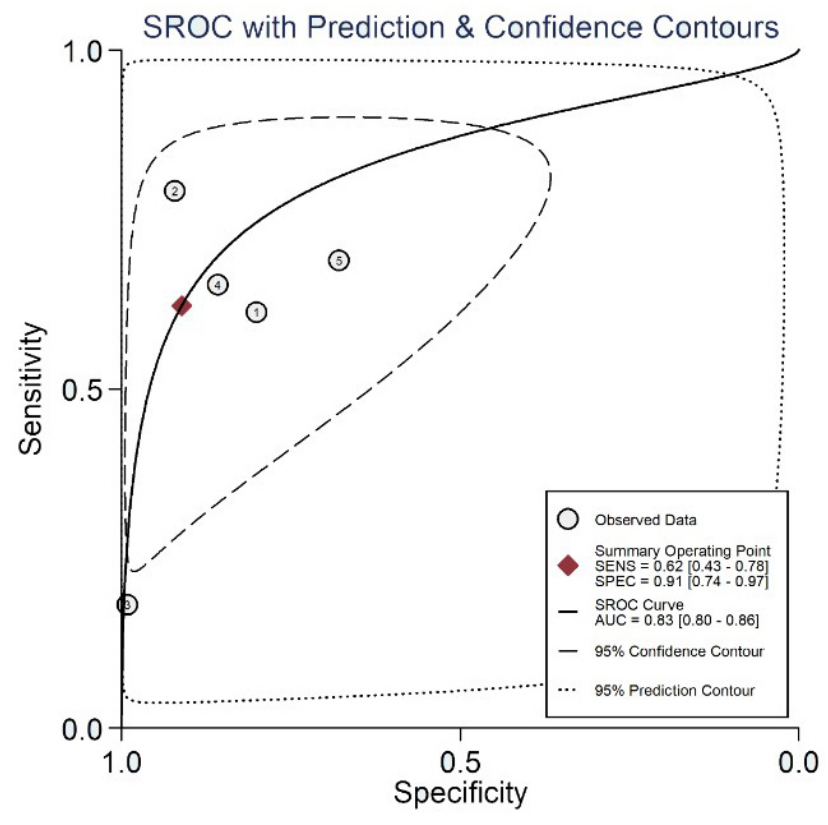

Fig. 4. SROC (Summary Receiver Operating Characteristic Curves) of the ultrasound with prediction $\&$ confidence contours.

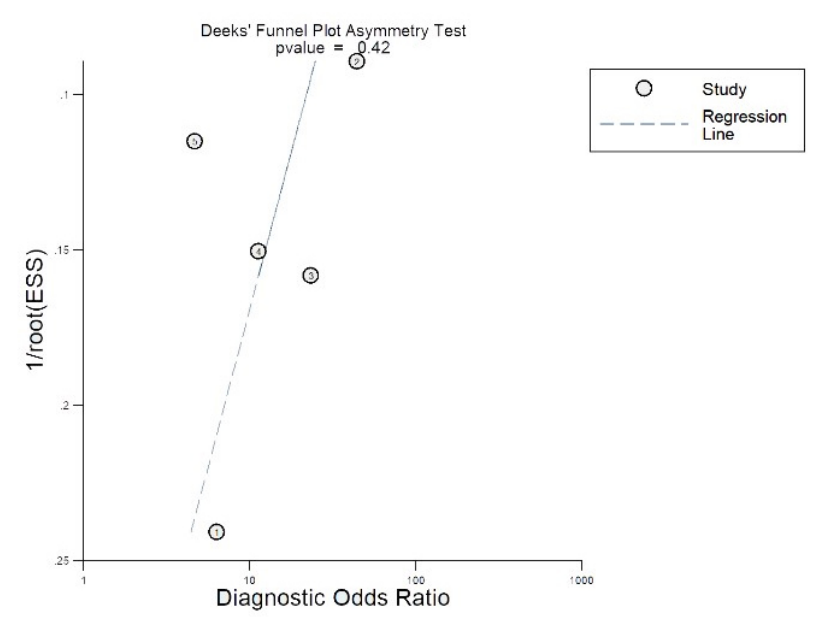

Fig. 5. Deeks' Funnel Plot Asymmetry Test.

Saini et al. [26], the technical cost of MRI was more expensive than that of ultrasound. The use of a class $C$ drug called gadolinium makes the safety of MRI uncertain according to the Food and Drug Administration [27]. Therefore, MRI is only used if the ultrasound results are inconclusive, for suspected appendicitis during pregnancy [27].

In this meta-analysis, the sensitivity and specificity for ultrasound in diagnosing of appendicitis during pregnancy were $62 \%$ and $91 \%$, respectively. It was reported that an optimized ultrasound algorithm significantly improved the diagnoses of appendicitis made by ultrasound, and the diagnosis performance might be further improved when operated by experienced radiologists [28,29]. Moreover, Le- ichtle and his colleagues [30] reported that obesity was associated with increased rate of incorrect results from ultrasound, and Hoffmann et al. [31] found that intra-abdominal air could foil image acquisition.

There are a few limitations in our study. All five articles included were retrospective studies. The five articles only included patients with suspected appendicitis that also underwent appendectomy. The information for pregnant patients who underwent ultrasound test but without the appendectomy was missing and no follow up information was obtained. All those factors might make the TP and FP lower than actual numbers. Moreover, those articles [32-36] provided the sensitivity and specificity values but not TP, FP, $\mathrm{TN}$ or FN values from the revision.

\section{Conclusions}

In summary, data of this meta-analysis revealed that ultrasound had a medium-level sensitivity and high specificity on the diagnosis of acute appendicitis in pregnant women.

\section{Author contributions}

YLL wrote the manuscript, conducted the statistical analyses, and retrieved data from medical database. SKL retrieved data and reviewed the final manuscript. All authors contributed to editorial changes in the manuscript. All authors read and approved the final manuscript.

\section{Ethics approval and consent to participate}

Not applicable.

\section{Acknowledgment}

We are very grateful to all people that helped us in the process of writing this manuscript. In addition, we would like to thank the anonymous reviewers who have helped to improve the paper.

\section{Funding}

This research received no external funding.

\section{Conflict of interest}

The authors declare no conflict of interest.

\section{References}

[1] Üçüncü MZ, Üçüncü MM. Left Sided Acute Appendicitis in Pregnant Woman: a Case Report. Turkish Journal of Colorectal Disease. 2016; 26: 136-138.

[2] Çınar H, Aygün A, Derebey M, Tarım İA, Akalın Ç, Büyükakıncak S, et al. Significance of hemogram on diagnosis of acute appendicitis during pregnancy. Turkish Journal of Trauma \& Emergency Surgery. 2018; 24: 423-428.

[3] Pastore PA, Loomis DM, Sauret J. Appendicitis in pregnancy. Journal of the American Board of Family Medicine. 2006; 19: 621-626.

[4] Beyazal M, Özkaçmaz S, Ünal Ö, Yavuz A, Bora A. Necrotizing 
Brucella epididymoorchitis: magnetic resonance imaging findings. Prevalence. 2014; 19: 24.

[5] Won RP, Friedlander S, Lee SL. Management and Outcomes of Appendectomy during Pregnancy. The American Surgeon. 2017; 83: 1103-1107.

[6] Aggenbach L, Zeeman GG, Cantineau AEP, Gordijn SJ, Hofker HS. Impact of appendicitis during pregnancy: no delay in accurate diagnosis and treatment. International Journal of Surgery. 2015; 15: 84-89.

[7] McGory ML, Zingmond DS, Tillou A, Hiatt JR, Ko CY, Cryer HM. Negative appendectomy in pregnant women is associated with a substantial risk of fetal loss. Journal of the American College of Surgeons. 2007; 205: 534-540.

[8] Lu Y, Friedlander S, Lee SL. Negative Appendectomy: Clinical and Economic Implications. The American Surgeon. 2016; 82: 1018-1022.

[9] Charfi S, Sellami A, Affes A, Yaïch K, Mzali R, Boudawara TS. Histopathological findings in appendectomy specimens: a study of 24,697 cases. International Journal of Colorectal Disease. 2014; 29: 1009-1012.

[10] Rosen MP, Ding A, Blake MA, Baker ME, Cash BD, Fidler JL, et al. ACR Appropriateness Criteria ${ }^{\circledR}$ right lower quadrant painsuspected appendicitis. Journal of the American College of Radiology. 2011; 8: 749-755.

[11] Lembcke B. Ultrasonography for acute appendicitis - the way it looks today. Zeitschrift Fur Gastroenterologie. 2016; 54: 11511165.

[12] Konrad J, Grand D, Lourenco A. MRI: first-line imaging modality for pregnant patients with suspected appendicitis. Abdominal Imaging. 2015; 40: 3359-3364.

[13] Tankel J, Yellinek S, Shechter Y, Greenman D, Ioscovich A, Grisaru-Granovsky S, et al. Delaying laparoscopic surgery in pregnant patients with an equivocal acute appendicitis: a stepwise approach does not affect maternal or fetal safety. Surgical Endoscopy. 2019; 33: 2960-2966.

[14] Baruch Y, Canetti M, Blecher Y, Yogev Y, Grisaru D, Michaan $\mathrm{N}$. The diagnostic accuracy of ultrasound in the diagnosis of acute appendicitis in pregnancy. The Journal of Maternal-Fetal \& Neonatal Medicine. 2019; 1-6.

[15] Whiting PF, Rutjes AW, Westwood ME, Mallett S, Deeks JJ, Reitsma JB, et al. QUADAS-2: a revised tool for the quality assessment of diagnostic accuracy studies. Annals of Internal Medicine. 2011; 155: 529-536.

[16] Aras A, Karaman E, Pekşen Ç, Kızıltan R, Kotan MÇ. The diagnosis of acute appendicitis in pregnant versus non-pregnant women: a comparative study. Revista Da Associacao Medica Brasileira. 2016; 62: 622-627.

[17] Segev L, Segev Y, Rayman S, Nissan A, Sadot E. The diagnostic performance of ultrasound for acute appendicitis in pregnant and young nonpregnant women: a case-control study. International Journal of Surgery. 2016; 34: 81-85.

[18] Eng KA, Abadeh A, Ligocki C, Lee YK, Moineddin R, AdamsWebber T, et al. Acute Appendicitis: a Meta-Analysis of the Diagnostic Accuracy of us, CT, and MRI as second-Line Imaging Tests after an Initial us. Radiology. 2018; 288: 717-727.

[19] Giljaca V, Nadarevic T, Poropat G, Nadarevic VS, Stimac D. Diagnostic Accuracy of Abdominal Ultrasound for Diagnosis of Acute Appendicitis: Systematic Review and Meta-analysis. World Journal of Surgery. 2017; 41: 693-700.

[20] Doria AS, Moineddin R, Kellenberger CJ, Epelman M, Beyene
$\mathrm{J}$, Schuh S, et al. Us or CT for Diagnosis of Appendicitis in Children and Adults? A Meta-Analysis. Radiology. 2006; 241: 8394.

[21] Kim HY, Park JH, Lee SS, Lee WJ, Ko Y, et al. CT in Differentiating Complicated From Uncomplicated Appendicitis: Presence of Any of 10 CT Features Versus Radiologists' Gestalt Assessment. American Journal of Roentgenology. 2019; 213: W218W227.

[22] Pickhardt PJ, Lawrence EM, Pooler BD, Bruce RJ. Diagnostic performance of multidetector computed tomography for suspected acute appendicitis. Annals of Internal Medicine. 2011; 154: 789-291.

[23] van Randen A, Bipat S, Zwinderman AH, Ubbink DT, Stoker J, Boermeester MA. Acute appendicitis: meta-analysis of diagnostic performance of $\mathrm{CT}$ and graded compression us related to prevalence of disease. Radiology. 2008; 249: 97-106.

[24] Yoon I, Slesinger TL. Radiation Exposure In Pregnancy. Treasure Island (FL): StatPearls Publishing. 2020.

[25] Kave M, Parooie F, Salarzaei M. Pregnancy and appendicitis: a systematic review and meta-analysis on the clinical use of MRI in diagnosis of appendicitis in pregnant women. World Journal of Emergency Surgery. 2019; 14: 37.

[26] Saini S, Seltzer SE, Bramson RT, Levine LA, Kelly P, Jordan PF, et al. Technical cost of radiologic examinations: analysis across imaging modalities. Radiology. 2000; 216: 269-272.

[27] Singh A, Danrad R, Hahn PF, Blake MA, Mueller PR, Novelline RA. MR imaging of the acute abdomen and pelvis: acute appendicitis and beyond. Radiographics. 2007; 27: 1419-1431.

[28] Chang ST, Jeffrey RB, Olcott EW. Three-step sequential positioning algorithm during sonographic evaluation for appendicitis increases appendiceal visualization rate and reduces CT use. American Journal of Roentgenology. 2014; 203: 1006-1012.

[29] Tepel J, Sommerfeld A, Klomp H, Kapischke M, Eggert A, Kremer B. Prospective evaluation of diagnostic modalities in suspected acute appendicitis. Langenbeck's Archives of Surgery. 2004; 389: 219-224.

[30] Leichtle S, Lucas JW, Kim WC, Aboutanos M. Decreasing Accuracy of the eFAST Examination-another Challenge Due to Morbid Obesity. The American Surgeon. 2019; 85: 923-926.

[31] Hoffmann B, Nürnberg D, Westergaard MC. Focus on abnormal air: diagnostic ultrasonography for the acute abdomen. European Journal of Emergency Medicine. 2012; 19: 284-291.

[32] Hiersch L, Yogev Y, Ashwal E, From A, Ben-Haroush A, Peled $Y$. The impact of pregnancy on the accuracy and delay in diagnosis of acute appendicitis. The Journal of Maternal-Fetal \& Neonatal Medicine. 2014; 27: 1357-1360.

[33] Shetty MK, Garrett NM, Carpenter WS, Shah YP, Roberts C. Abdominal computed tomography during pregnancy for suspected appendicitis: a 5-year experience at a maternity hospital. Seminars in Ultrasound, CT and MRI. 2010; 31: 8-13.

[34] Aggenbach L, Zeeman GG, Cantineau AEP, Gordijn SJ, Hofker HS. Impact of appendicitis during pregnancy: no delay in accurate diagnosis and treatment. International Journal of Surgery. 2015; 15: 84-89.

[35] Kazim SF, Inam Pal KM. Appendicitis in pregnancy: Experience of thirty-eight patients diagnosed and managed at a tertiary care hospital in Karachi. International Journal of Surgery. 2009; 7: $365-367$.

[36] Lemieux P, Rheaume P, Levesque I, Bujold E, Brochu G. Laparoscopic appendectomy in pregnant patients: a review of 45 cases. Surgical Endoscopy. 2009; 23: 1701-1705. 